\title{
A Rat Model with Multivalve Calcification Induced by Subtotal Nephrectomy and High-Phosphorus Diet
}

\author{
Liting Wang ${ }^{a, b}$ Rining Tang ${ }^{a, b}$ Yuxia Zhang ${ }^{a, b}$ Zixiao Liu ${ }^{c}$ Sijie Chen ${ }^{a, b}$ \\ Kaiyun Song ${ }^{\mathrm{a}, \mathrm{b}}$ Yu Guo ${ }^{\mathrm{a}, \mathrm{b}}$ Li Zhang $^{\mathrm{a}} \quad$ Xiaochen Wang $^{\mathrm{a}}$ Xiaobin Wang $^{\mathrm{d}}$ \\ Hong Liu ${ }^{a}$ Xiaoliang Zhang ${ }^{a}$ Bi-Cheng Liu ${ }^{a}$ \\ anstitute of Nephrology, Zhong Da Hospital, School of Medicine, Southeast University, Nanjing, China; \\ ${ }^{b}$ Institute of Nephrology, NanJing LiShui People's Hospital, Zhongda Hospital Lishui Branch, School of Medicine, \\ Southeast University, Nanjing, China; 'State Key Laboratory for Modification of Chemical Fibers and Polymer \\ Materials, International Joint Laboratory for Advanced Fiber and Low-Dimension Materials, College of Materials \\ Science and Engineering, Donghua University, Shanghai, China; ${ }^{\mathrm{d}}$ Experimental Animal Centers School of Medicine, \\ Southeast University, Nanjing, China
}

\section{Keywords}

Valve calcification · Chronic kidney disease .

5/6 nephrectomy · Phosphorus

\begin{abstract}
Background: Chronic kidney disease (CKD) with known valve calcification (VC) places individuals at high risk of cardiovascular disease. The study of VC in CKD is challenging due to the lack of a suitable research model. Here, we established a rat model of multivalve calcification induced by subtotal nephrectomy and a high-phosphate (HP) diet and analyzed the valve characteristics. Methods: We established a CKD model in Sprague-Dawley rats by performing 5/6 nephrectomy $(5 / 6 \mathrm{Nx})$ followed by feeding with chow containing different phosphate concentrations for 8,12 , or 16 weeks. The rats were divided into 4 groups: sham+normal phosphate (NP, 0.9\% P), sham+high phosphate (HP, 2.0\% P), $5 / 6 \mathrm{Nx}+\mathrm{NP}$, and $5 / 6 \mathrm{~N} x+\mathrm{HP}$. Serum creatinine $(\mathrm{Scr})$, blood
\end{abstract}

\section{(C) 2020 The Author(s)}

Published by S. Karger AG, Basel

Karger

Open access

This article is licensed under the Creative Commons AttributionNonCommercial-NoDerivatives 4.0 International License (CC BYNC-ND) (http://www.karger.com/Services/OpenAccessLicense). Usage and distribution for commercial purposes as well as any distribution of modified material requires written permission. urea nitrogen (BUN), parathyroid hormone (PTH), calcium, phosphorus, and 24-h urine protein levels were investigated. Pathological examinations included histological characterization, safranin staining, Alcian blue staining, and von Kossa staining at different time points. Using nanoanalytical electron microscopy, we examined valves from rats in the $5 / 6 \mathrm{Nx}+\mathrm{HP}$ and sham $+\mathrm{HP}$ groups and detected spherical particles using energy-dispersive spectroscopy (EDS) to observe microscopic changes in the valves. In addition, the calcified tissues were analyzed for phase and crystallization properties using an X-ray powder diffractometer. Results: The rats in the $5 / 6 \mathrm{Nx}+\mathrm{HP}$ and $5 / 6 \mathrm{Nx}+\mathrm{NP}$ groups presented with increased levels of Scr, BUN, and 24-h urine protein compared with those of the rats in the sham+HP and sham+NP groups. High levels of PTH were observed, and hematoxylin and eosin staining and immunohistochemistry for proliferating cell nuclear antigen showed parathyroid hyperplasia in rats in the $5 / 6 \mathrm{Nx}+\mathrm{HP}$ group but not in the $5 / 6 \mathrm{Nx}+\mathrm{NP}$ group. In rats in the $5 / 6 \mathrm{Nx}+\mathrm{HP}$ group, extracellular matrix glycosylation 
was observed in the aortic valve in the 12th week and the mitral valve in the 16th week. In the 16th week, chondrocytes appeared in the aortic valve, as confirmed by immunofluorescence and Western blotting. Calcified particles mainly composed of phosphorus and calcium were observed in both the aortic and mitral valves by transmission electron microscopy and scanning electron microscopy (SEM). The main mineral component of the calcified aortic valve particles was hydroxyapatite $\left[\mathrm{Ca}_{5}\left(\mathrm{PO}_{4}\right)_{3}(\mathrm{OH})\right]$, as shown by X-ray diffraction. However, there were no obvious differences in heart function between rats in the $5 / 6 \mathrm{Nx}+\mathrm{HP}$ and sham $+\mathrm{HP}$ groups. Conclusions: Our findings demonstrate that multivalve calcification is involved in CKD following 16-week HP and that hydroxyapatite $\left[\mathrm{Ca}_{5}\left(\mathrm{PO}_{4}\right)_{3}(\mathrm{OH})\right]$ is the main component of the calcified aortic valve particles of rats in the $5 / 6 \mathrm{Nx}+\mathrm{HP}$ group.

(c) 2020 The Author(s)

Published by S. Karger AG, Basel

\section{Introduction}

Ectopic calcification involving deposition of calcium phosphate crystals is a frequent chronic kidney disease (CKD) complication and includes vascular calcification [1], valve calcification (VC) [2], and skin calcification [3]. Vascular calcification and VC involved an active process in which vascular smooth muscle cells or valve interstitial cell undergo a phenotypic transformation into osteoblast-like cells, similar in many ways to bone metabolism $[2,4]$. VC and vascular calcification are common complications of CKD and critical indicators of cardiovascular disease (CVD) and all-cause mortality in patients with CKD [5-7] or end-stage renal disease [8]. According to the guidelines published in Kidney Disease: Improving Global Outcomes (KIDGO), CKD patients with known $\mathrm{VC}$ are at the highest risk of CVD [9]. The incidence of VC increases with CKD progression, with an incidence of approximately $40 \%$ in patients with stage 3 CKD and 80 $99 \%$ in patients with stage 5 CKD [10-14]. However, basic studies of CKD VC are challenging due to the lack of a suitable research model.

In a previous study, Shuvy et al. [15] used a high-adenine $(0.75 \%)$ and high-phosphate (HP) diet (1.5\%) for 7 weeks to establish an aortic VC model. The short duration of VC clearly did not coincide with the duration of VC observed in CKD patients in the clinic, and the renal function of this model was recoverable. In addition, single heart valves were typically not affected, and the aortic (45-59\%) and mitral (34-55\%) valves were most frequently involved [16]. Most current studies focus on vas- cular calcification. In the animal model of $\mathrm{CKD}$ vascular calcification, the vascular calcium $(\mathrm{Ca})$ content was more than twice that of normal blood vessels [17]. Hence, we planned to establish a stable uremic model that is more compatible with the clinical findings in kidney dialysis patients and assess changes in the aortic and mitral valves.

Commonly used methods for CKD include surgery (5/6 nephrectomy, 5/6Nx) [18], electrocautery [19], and drug induction (adenine [20], oxalate [21]). The well-established $5 / 6 \mathrm{Nx}$ model used in our laboratory mimics human CKD with increased serum creatinine (Scr) levels [22] and tubulointerstitial fibrosis [18]. Following $5 / 6 \mathrm{Nx}$, increased levels of phosphate in the diet could induce parathyroid hormone (PTH) secretion, which causes disordered $\mathrm{Ca}$ and phosphorus metabolism that accelerates VC. Commonly used concentrations of phosphate in the diet are 1.2 [23] and 1.8\% [24]. Recently, we developed a rat model of bone abnormalities in CKD induced by a diet containing adenine and $1.8 \%$ phosphate [20]. However, in our study and other studies, there was no obvious VC among rats fed a $1.8 \%$ phosphate diet, as shown by microcomputed tomography (micro-CT) examination [25]. Hence, increasing the concentration of phosphate in the diet for 8 weeks might induce VC. Valve cells express higher levels of anti-inflammatory mRNA than do vascular cells [26], indicating that the 8-week duration of the HP diet was not sufficient. Therefore, we need to determine the optimal concentration of phosphate in the HP diet administered for different lengths of time.

Hence, in this study, we examined multivalve calcification in a $5 / 6 \mathrm{Nx}$ and HP-fed CKD rat model. This study aimed to determine the contribution of an HP diet to long-term multivalve calcification for different lengths of time in a CKD model, which is more compatible with the clinical findings in kidney dialysis patients than other CKD models.

\section{Methods}

Animals

Eight-week-old male Sprague-Dawley rats (Animal Laboratory of Nantong University, China) $(n=40)$ were fed a normal phosphate (NP) diet $(0.9 \% \mathrm{P}$ and $1.0 \% \mathrm{Ca})$ (Nanjing, China) and divided into four experimental groups (Fig. 1): (A) sham $+\mathrm{HP}(n=$ $10)$; (B) sham $+\mathrm{NP}(n=10)$; (C) $5 / 6 \mathrm{Nx}+\mathrm{HP}(n=20)$; and (D) $5 / 6 \mathrm{Nx}+\mathrm{NP}(n=10)$. The concentrations of phosphorus in the chow fed to the rats in each group were $2.0,0.9,2.0$, and $0.9 \%$. The $5 / 6 \mathrm{Nx}$ procedure was performed as previously described [18]. All animals received humane care, and the study protocols complied with institutional guidelines. 
Table 1. Serum and urine biochemical and physiological parameters in the $5 / 6 \mathrm{Nx}$ and sham groups

\begin{tabular}{lcrcc}
\hline & Sham $+\mathrm{NP}$ & Sham $+\mathrm{HP}$ & $5 / 6 \mathrm{Nx}+\mathrm{NP}$ & $5 / 6 \mathrm{Nx}+\mathrm{HP}$ \\
\hline Scr, $\mu \mathrm{mol} / \mathrm{L}$ & $67.33 \pm 11.59$ & $54.69 \pm 8.96$ & $413.38 \pm 24.01^{*}$ & $367.78 \pm 44.35^{*}$ \\
BUN, mmol/L & $0.63 \pm 0.1$ & $0.74 \pm 0.06$ & $2.71 \pm 0.69^{*}$ & $2.58 \pm 0.64^{*}$ \\
24-h urine protein, mg & $13.78 \pm 1.27$ & $13.9 \pm 1.27$ & $280.02 \pm 41.53^{*}$ & $255.16 \pm 39.89^{*}$ \\
PTH, pg/mL & $66.67 \pm 8.99$ & $67.83 \pm 5.97$ & $81.17 \pm 14.27$ & $411.60 \pm 26.61^{*, \#}$ \\
$\mathrm{Ca}, \mathrm{mmol} / \mathrm{L}$ & $1.33 \pm 0.086$ & $1.06 \pm 0.08$ & $1.087 \pm 0.02$ & $1.146 \pm 0.17$ \\
P, mmol/L & $2.67 \pm 0.24$ & $2.91 \pm 0.58$ & $2.66 \pm 0.43$ & $4.61 \pm 0.65^{*, \#}$ \\
\hline
\end{tabular}

BUN, blood urea nitrogen; PTH, parathyroid hormone; $\mathrm{P}$, phosphate; $\mathrm{Ca}$, calcium. The data are represented as the means \pm SEMs $(n=8) .{ }^{*} p<0.05$ vs. sham $+\mathrm{HP}$ and sham + NP by ANOVA. ${ }^{*} p<0.05$ vs. $5 / 6 \mathrm{Nx}$ by ANOVA.

\section{Serum Biochemistry}

Blood urea nitrogen (BUN), Scr, Ca, phosphorus (P), and 24-h urine protein levels were measured by a semiautomatic biochemical analyzer (ECA-2000A, Jilin, China) and a UV-5100 spectrophotometer (Shanghai, China). Serum PTH levels were determined via ELISA (Abnova).

\section{Histology}

For valve histopathology, the rat valves were isolated and carefully removed with a scalpel. Tissue samples for histological analysis were fixed in $10 \%$ phosphate-buffered formalin. The tissues were embedded in paraffin, sectioned, and stained with hematoxylin and eosin (HE), safranin green, Alcian blue, and von Kossa according to standard methods [27].

\section{Western Blotting}

The protein samples were separated by sodium dodecyl sulfatepolyacrylamide gel electrophoresis and then semiwet transferred onto polyvinylidene fluoride membranes. The nonspecific binding sites were blocked with $5 \%$ bovine serum albumin (BSA) dissolved in Tris-buffered saline containing $0.1 \%$ Tween 20 for $1 \mathrm{~h}$ at room temperature (RT). The membranes were incubated with primary antibodies against Sox9 (ab185966) and $\alpha$-actin (ab179467) overnight at $4{ }^{\circ} \mathrm{C}$. Peroxidase-conjugated secondary antibodies were incubated with the membranes for $1 \mathrm{~h}$. The bands were detected using a chemiluminescent horseradish peroxidase substrate (Merck Millipore) with an ImageQuant LAS 4000 image acquisition system.

\section{Immunofluorescence Staining}

The cryosections $(5 \mu \mathrm{m})$ were fixed with $4 \%$ paraformaldehyde, permeabilized with $0.25 \%$ Triton X-100, and blocked with 5\% BSA in phosphate-buffered saline at RT. The slides were then immunostained with the primary antibody against Sox9 (ab185966) at $4{ }^{\circ} \mathrm{C}$ overnight. After incubation with the secondary antibody for $1 \mathrm{~h}$ in the dark at RT, images were captured using a laser scanning confocal microscope (LSM 710 META, Zeiss, Germany).

\section{Immunohistochemistry}

The paraffinized sections were dewaxed in water, and the dewaxed, hydrated tissue sections were pretreated with an antigen retrieval solution ( $\mathrm{pH}$ 6.0). The sections were incubated with $3 \%$ $\mathrm{H}_{2} \mathrm{O}_{2}$ in deionized water for 15 min to block endogenous peroxidase and rinsed with PBS. Antibodies against ICAM-1 (1:400) and proliferating cell nuclear antigen (PCNA) (ab92552, 1:400) were

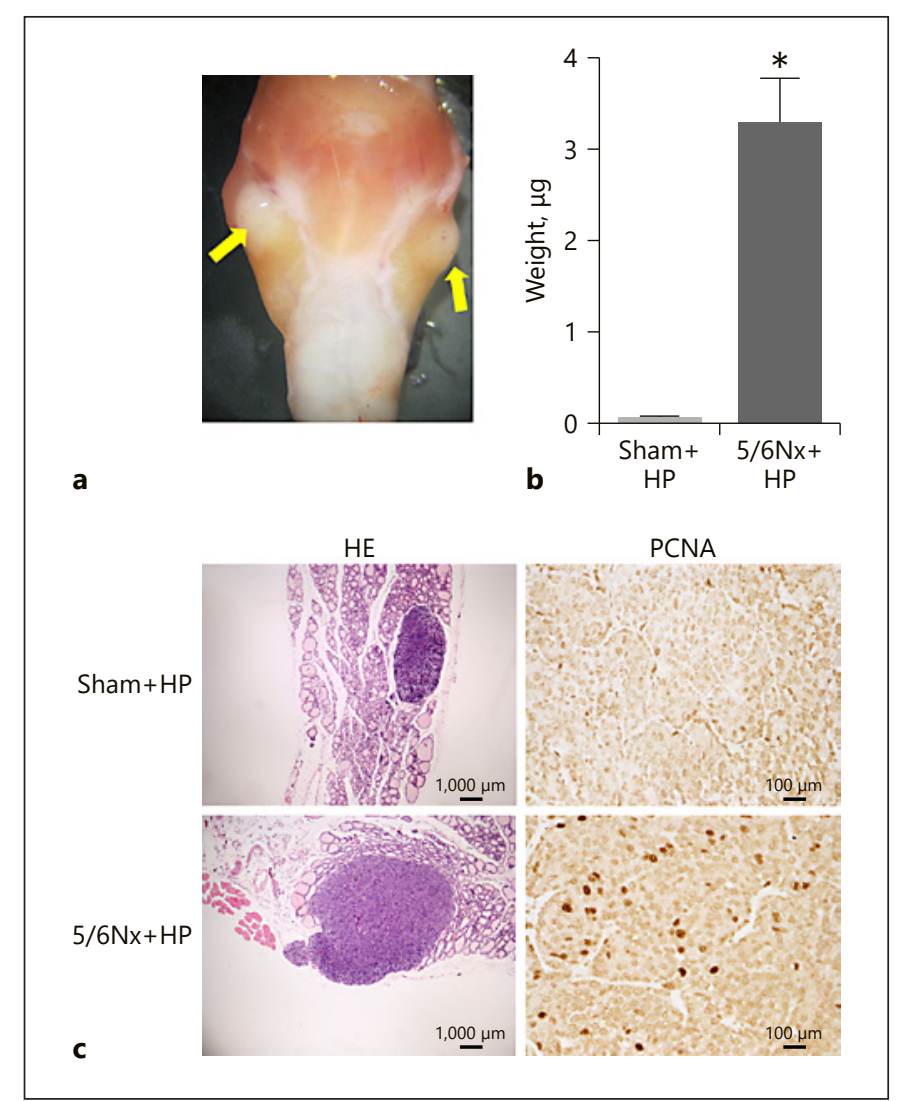

Fig. 1. Parathyroid enlargement of the $5 / 6 \mathrm{Nx}+\mathrm{HP}$ group. a The general parathyroid performance of the $5 / 6 \mathrm{Nx}+\mathrm{HP}$ group. $\mathbf{b}$ The increased weight of the parathyroid in the $5 / 6 \mathrm{Nx}+\mathrm{HP}$ group compared with that in the sham $+\mathrm{HP}$ group. c Comparison of HE staining and PCNA expression between the $5 / 6 \mathrm{Nx}+\mathrm{HP}$ and sham $+\mathrm{HP}$ groups.

added, and the sections were incubated overnight at $4{ }^{\circ} \mathrm{C}$. The cells were immersed in PBS, and the IgG antibody Fab fragment-HRP polymer was added. The mixture was then incubated for $30 \mathrm{~min}$ at RT $\left(37^{\circ} \mathrm{C}\right)$, followed by washing 5 times with PBS for 3 min each. Color was developed using a DAB solution. 
Echocardiographic Assessment of Left Ventricular Function

After being fed the HP diet for 16 weeks, the rats were anesthetized via the continuous administration of $2 \%$ isoflurane in $100 \%$ oxygen at $2 \mathrm{~L} / \mathrm{min}$ via a facemask. Then, transthoracic echocardiography was performed in all rats using an ultrasound system (Vevo 2100, VisualSonics Inc., Toronto, ON, Canada) with a 21$\mathrm{MHz}$ probe. The left ventricular ejection fraction and fractional shortening were calculated to evaluate left ventricle function. Measurements over three consecutive cardiac cycles were averaged. A long-axis M-mode view was adopted when analyzing the aortic diameter, and Doppler echocardiography was used to determine the velocity and pressure gradient in the heart. Data analysis was performed in a blinded fashion.

\section{Electron Microscopy of the Valve Microstructure}

Fresh and decellularized aortic valve leaflet samples were fixed in a mixture of $2.5 \%$ glutaraldehyde and $4 \%$ paraformaldehyde at $\mathrm{pH} 7.35$ for $30 \mathrm{~min}$ at RT for either scanning electron microscopy (SEM) (S-4800, Hitachi, equipped with energy dispersive spectroscopy [EDS]) or transmission electron microscopy (TEM) (HRTEM, FEI Talos F200S, equipped with EDS). After fixation and dehydration with ethanol, the samples were embedded in Durcupan resin for ultrathin-sectioning and ultramicroscopy observation. EDS was performed with a TEM and SEM operating at 200 $\mathrm{kV}$, which provided spatial resolutions of $500 \mathrm{~nm}$ and $30 \mu \mathrm{m}$, respectively.

\section{X-Ray Diffraction}

The calcified tissues were analyzed for phase and crystallization properties using an X-ray powder diffractometer. The calcified area of the heart valve was removed under a stereoscopic microscope, washed three times with distilled water, and dried using a freeze-drying vacuum system. Powder X-ray diffraction (XRD) measurements were made on a Bruker D4 X-ray diffractometer with $\mathrm{Cu} \mathrm{Ka}$ radiation at a scan rate of $0.05(2 \theta) \mathrm{s}^{-1}(\lambda=0.15418$ $\mathrm{nm})$. The dried powder was evenly spread on the surface of the slide and placed on the stage of an X-ray powder diffractometer. The instrument used copper as the target, with Ka-ray $(\lambda=1.54$ $\mathrm{nm}$ ) as the radiation source, a tube voltage of $40 \mathrm{kV}$, a tube current of $35 \mathrm{~mA}$, and a scan speed of $5^{\circ} / \mathrm{min}$; a $2 \theta 10 \sim 90^{\circ}$ diffraction intensity curve was recorded.

\section{Statistical Analysis}

All results were expressed as the mean \pm standard deviation. If the data conformed to the parametric test, then the differences among groups were compared by the Bonferroni test for post hoc analysis after one-way ANOVA test. Otherwise, a nonparametric test (such as the rank-sum test) was used for analysis by SPSS 21.0 statistical software. Differences with $p$ values $<0.05$ were considered statistically significant.

\section{Results}

\section{Renal Function and Biochemical Parameters}

The 8-week-old Sprague-Dawley male rats were randomly allocated to the following experimental groups: control groups, which consisted of the sham plus NP
$($ sham $+\mathrm{NP})$ group and the sham plus high phosphate $($ sham $+\mathrm{HP}$ ) group, and the $5 / 6 \mathrm{Nx}$ groups, which consisted of $5 / 6 \mathrm{Nx}$ plus normal phosphorus $(5 / 6 \mathrm{Nx}+\mathrm{NP})$ group and the $5 / 6 \mathrm{Nx}$ plus high phosphate $(5 / 6 \mathrm{Nx}+\mathrm{HP})$ group.

The BUN, Scr, and 24-h urine protein levels were significantly higher in the CKD group than in the control group, but no difference was observed between the $5 / 6 \mathrm{Nx}+\mathrm{HP}$ and $5 / 6 \mathrm{Nx}+\mathrm{NP}$ groups (Table 1 ). The BUN and Scr levels were increased in the CKD group compared to the control group. Although serum phosphorus levels were comparable among the $5 / 6 \mathrm{Nx}+\mathrm{NP}$ and sham groups, we identified an approximately 2 -fold increase in serum phosphorus levels among rats in the $5 / 6 \mathrm{Nx}+\mathrm{HP}$ group relative to serum phosphorus levels in all other groups. Serum Ca levels were comparable among the groups. The $5 / 6 \mathrm{Nx}+\mathrm{HP}$ group also exhibited a 5 -fold increase in $\mathrm{PTH}$ levels compared to those in the sham and $5 / 6 \mathrm{Nx}+\mathrm{NP}$ groups.

\section{Parathyroid Hyperplasia}

Gross observation, weight measurements, and HE staining showed that the weight of the parathyroid gland was significantly greater in the $5 / 6 \mathrm{Nx}+\mathrm{HP}$ group $(3.26 \pm$ $0.49 \mu \mathrm{g})$ than in the control group $(0.04 \pm 0.01 \mu \mathrm{g})(p<$ 0.05 ) (Fig. 1). Consistent with parathyroid enlargement, immunohistochemistry demonstrated the upregulated expression of PCNA in rats in the $5 / 6 \mathrm{Nx}+\mathrm{HP}$ group.

\section{Valve Changes after Different Lengths of Time on an HP Diet}

Both safranin green and Alcian blue staining showed that the aortic valves of rats fed an HP diet for 12 weeks showed more extracellular matrix (ECM) glycosylation, a dominant hallmark of early $\mathrm{VC}$, than did those of rats fed an HP diet for 8 weeks (Fig. 2a). After the rats were fed an HP diet for 16 weeks, the appearance of chondrocytes was confirmed by immunofluorescence and Western blotting analysis (Fig. 2c, d). Additionally, severe VC was observed by von Kossa staining (Fig. 2a). However, ECM glycosylation appeared later in the mitral valve than in the aortic valve, and no chondrocytes were observed after the rats were fed the HP diet for 16 weeks (Fig. 2b).

\section{Microchanges in the Aortic and Mitral Valves}

To further clarify microchanges in the heart valves, we performed TEM and SEM. First, the aortic mitral valves were examined with SEM to visualize the topographical characteristics of the tissue. The surfaces of the normal aortic valves did not contain macroscopically observable calcified lesions. Although there were no obvious macro- 
HE
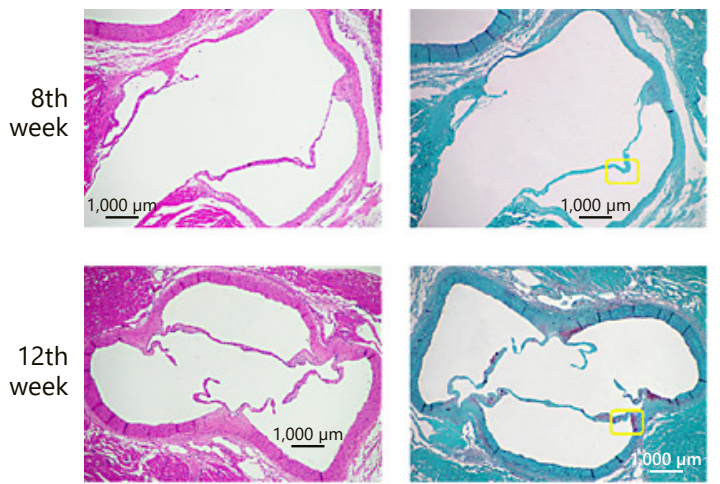

HE
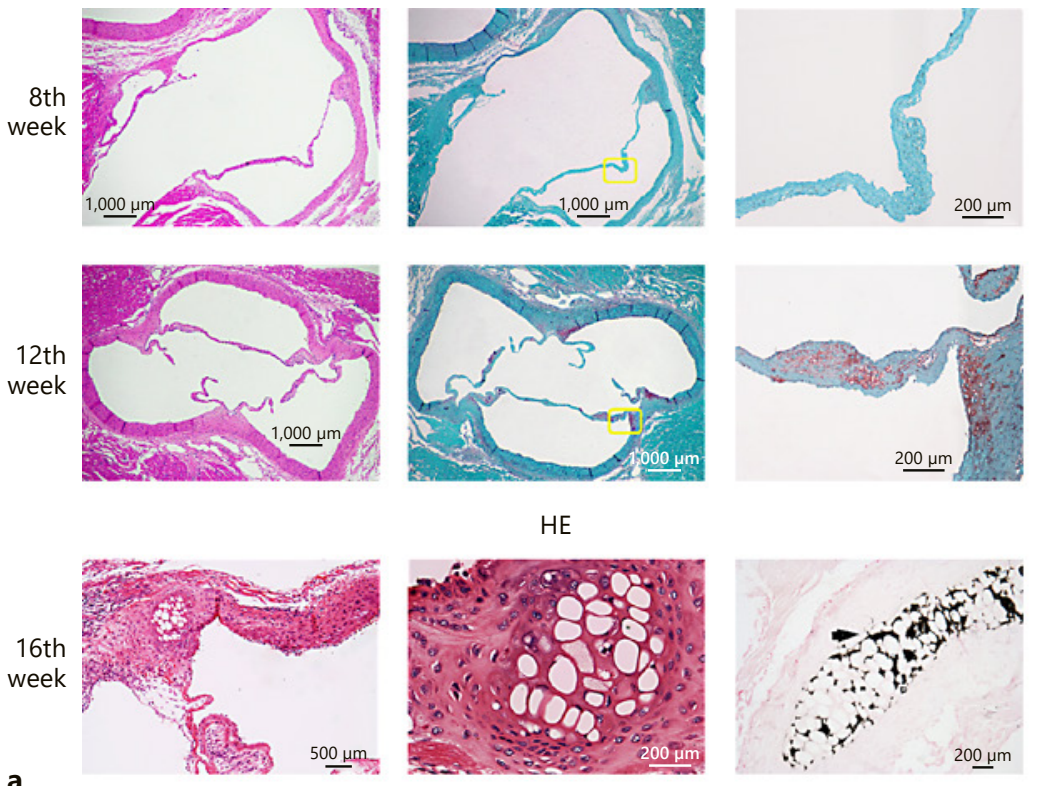

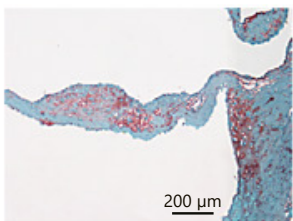

Alcian blue staining
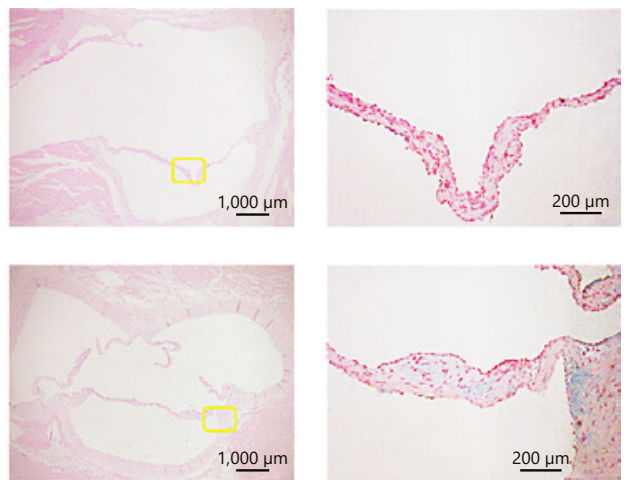

Von Kossa
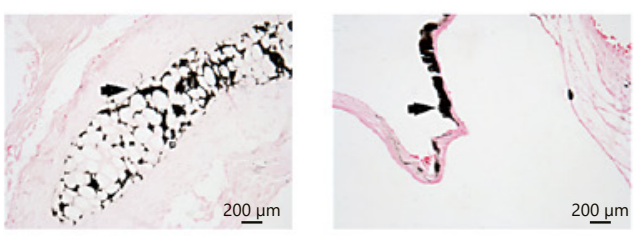

HE
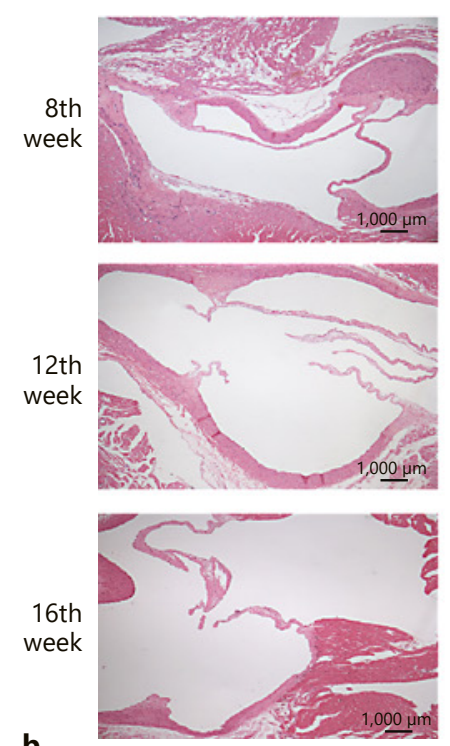

Mitral valve changes of $5 / 6 \mathrm{Nx}$ group Safranin green staining
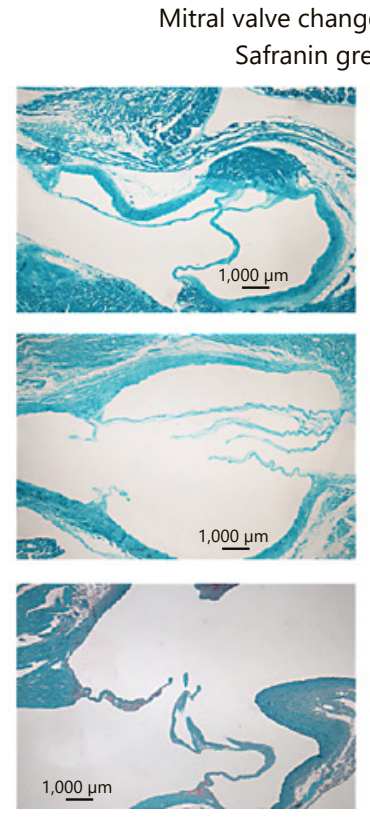
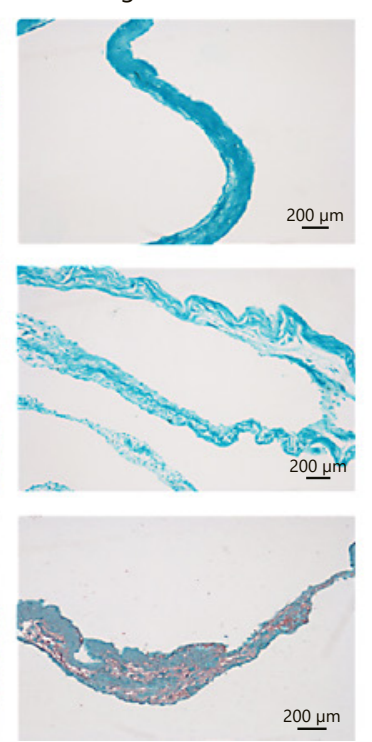

$\underline{200 \mu \mathrm{m}}$
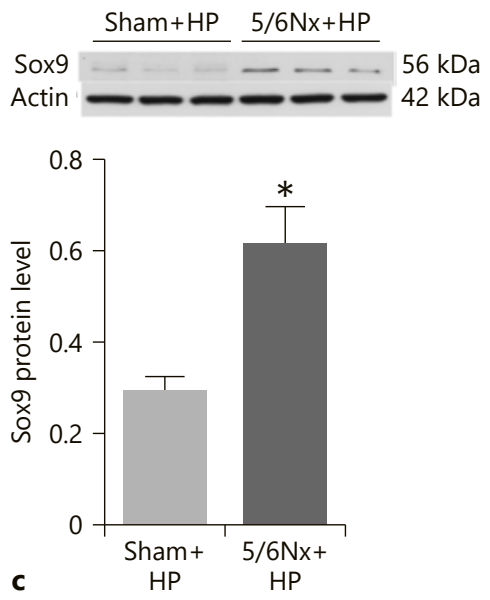

d

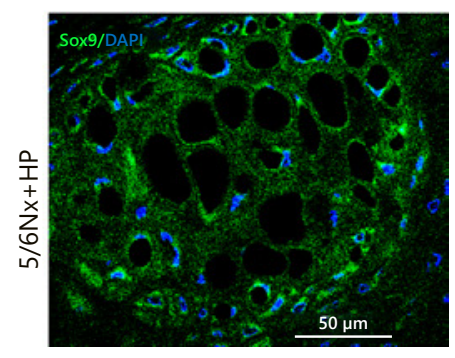

Fig. 2. Changes in the aortic and mitral valves of the $5 / 6 \mathrm{Nx}+\mathrm{HP}$ group after 8,12 , and 16 weeks. a ECM glycosylation of the aortic valve in the 12th week that was not observed in the 8th week (yellow box). b ECM glycosylation of the mitral valve in the 16th week that was not observed in the 8th and 10th weeks. Chondrocytes appeared in the 16th week (a) and were confirmed by von Kossa staining (black arrow), immunofluorescence (c), and Western blotting analysis (d). 
Fig. 3. SEM images, TEM images, EDS mapping, and XRD analyses of aortic valve tissues. a, b The samples from the $5 / 6 \mathrm{Nx}+\mathrm{HP}$ group were obtained from valves without macroscopic calcified lesions. The surfaces of the aortic and mitral valves were free of macroscopically observable calcified lesions but presented spherical particles. c High-angle annular dark field TEM image of spherical particles containing calcium and phosphorus on the surface of a valve without macroscopic calcified lesions. EDS and XRD demonstrated that macroscopic aortic valve calcified lesions (d) are composed of hydroxyapatite (e).

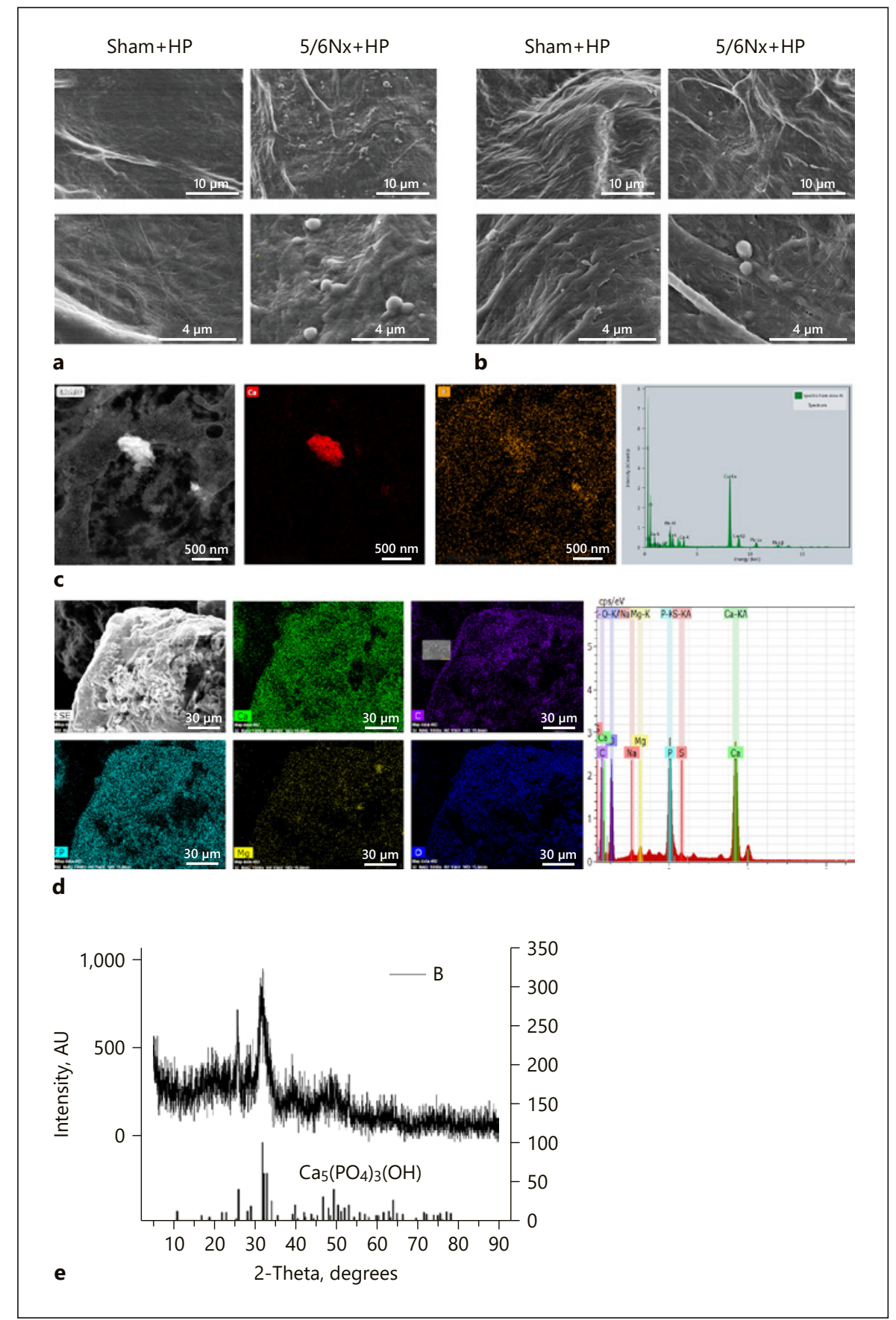

scopic calcified lesions in the mitral valves, numerous spherical particles were found in both the aortic and mitral valves of the $5 / 6 \mathrm{Nx}+\mathrm{HP}$ group (Fig. $3 \mathrm{a}, \mathrm{b}$ ). To identify the chemical elements constituting these particles, we performed EDS by TEM. The particles contained abundant $\mathrm{Ca}$ and phosphorus with small amounts of magnesium (Fig. 3c). The same composition was observed in obvious aortic VC deposits (Fig. 3d), and elemental analysis by EDS established that the material was entirely organic due to an abundance of oxygen and carbon. To clarify the specific components of the VC deposits, we performed XRD, which suggested that the calcified deposits were hydroxyapatite (Fig. 3e). 
Differences in Heart Function between the 5/6Nx+HP and Sham + HP Groups

Although there were obvious differences in the valves of rats in the $5 / 6 \mathrm{Nx}+\mathrm{HP}$ and sham $+\mathrm{HP}$ groups, we measured heart function in all experimental rats in the 4 groups but did not find any differences. These results are shown in the supplementary data (S1; for all online suppl. material, see www.karger.com/doi/10.1159/000506013).

\section{Other Heart Pathologies Caused by High Phosphorus}

Levels

Gross observation (S2A) and von Kossa staining showed coronary calcification in the $5 / 6 \mathrm{Nx}+\mathrm{HP}$ group that was not observed in the sham $+\mathrm{HP}$ group. In addition, $\mathrm{HE}$ and Sirius red staining of dead $5 / 6 \mathrm{Nx}+\mathrm{HP}$ rats showed myocardial infarction with cell-free, elliptical mass-like characteristics (S2C-E).

\section{Discussion}

Patients with advanced CKD are extremely vulnerable to severe VC, which presents high risks for adverse CVD outcomes, such as congestive heart failure and myocardial infarction $[28,29]$. Although there is a high incidence of heart VC among CKD G3-G5 patients [30], our understanding of $\mathrm{VC}$ is far from satisfactory. The study of CKD $\mathrm{VC}$ is challenging due to the lack of a suitable research model. Hence, the development of animal models of VC is of vital importance to facilitate research on the cardiovascular system.

We prolonged the formation of the rat model to 16 weeks to better simulate the long course of CKD mineral and bone disorder. At least 1 month is needed for animal models of CKD to display steady disease characteristics after intervention (e.g., after $5 / 6 \mathrm{Nx}$ or adenine administration). Short-term rat models cannot serve as reliable models of valve changes. Considering these factors, we prolonged the study duration. We observed elevated serum PTH levels and parathyroid hyperplasia in our rat model. In addition, an advantage of our rat model is that it best simulates the human disease condition.

To develop a severe VC model, we performed $5 / 6 \mathrm{Nx}$ and fed rats an HP diet in which the concentration of $\mathrm{P}$ (phosphate) was increased to $2.0 \%$, which was higher than that used in previous studies. Valve changes in CKD were determined after different lengths of time compared to those used in previous studies. Upon hyperphosphatemia, glycosylation of aortic valve leaflets and the mitral valve was observed in the 12th and 16th weeks, respec- tively, by safranin green and Alcian blue staining. Unlike the mitral valve, the aortic valve exhibited chondrocytelike cells in the 16th week, and aortic VC was confirmed by von Kossa staining. Although no obvious calcification was observed in the mitral valve, we examined the valves by nanoanalytical electron microscopy techniques combined with EDS. Spherical calcium phosphate particles were detected in the aortic and mitral valves of the $5 / 6 \mathrm{Nx}+\mathrm{HP}$ group but not in those of the $5 / 6 \mathrm{Nx}+\mathrm{NP}$ group. Calcium phosphate particles, which were first described in 2013 [31], play a fundamental role in calcified lesion formation. In our study, calcium phosphate particles were located in the fibrosa layer, which is proposed to be the point of calcified nodule initiation. To further clarify the components of VC deposits, we performed XRD. By comparison to standards, we found that the main component of aortic valve calcified lesions was hydroxyapatite $\left[\mathrm{Ca}_{5}\left(\mathrm{PO}_{4}\right)_{3}(\mathrm{OH})\right]$, which is consistent with the composition of human heart VC deposits.

We simultaneously used echocardiography to evaluate aortic valve and mitral valve function in the rat model. However, there were no significant differences in valve function between the rats in the $5 / 6 \mathrm{Nx}$ group and those in the sham group. In addition to valvular calcification, myocardial calcification and coronary artery calcification were observed in the rat model, which coincided with the observations of clinical autopsy [32]. Notably, myocardial infarction that may be related to myocardial ischemia occurred in $50 \%$ of the rats after the 16th week.

Taken together, our findings demonstrate the involvement of multivalve calcification in CKD following 16 weeks of exposure to HP. Unlike in previous studies, heart VC was evaluated by techniques ranging from specific pathological staining to microstructure analysis and from component analysis to elemental analysis to determine changes in multiple cardiac valves. This model may be useful for exploring CKD-related heart injury.

\section{Acknowledgement}

Thanks are due to BC Liu for valuable discussion.

\section{Statement of Ethics}

Animal experiments conform to internationally accepted standards and have been approved by the appropriate institutional review body. 


\section{Disclosure Statement}

There is no conflict of interest among all authors.

\section{Funding Sources}

This study was funded by grants from the National Natural Science Foundation of China (81770735,31571186), The Medical Talents of Jiangsu Province (ZDRCA2016079), Postgraduate Research and Practice Innovation Program of Jiangsu Province (KYCX_180183), Jiangsu Commission of Health (LGY2018097).

\section{Author Contributions}

R.N. Tang: This author is the co-first author and takes responsibility for all aspects of the reliability and freedom from bias of the data presented and their discussion.

L.T. Wang and R.N. Tang designed the study, performed the experiments, analyzed the results, and wrote and edited the manuscript. K.Y. Song, Y. Guo, X.C. Wang, and S. Chen performed the experiments and discussed the results. Z.X. Liu, X.B. Wang, L. Zhang, and Y.X. Zhang performed the experiments and analyzed the data. H. Liu, X.L. Zhang, and B.C. Liu reviewed and edited the manuscript.

\section{References}

1 Shanahan CM, Crouthamel MH, Kapustin A, Giachelli CM. Arterial calcification in chronic kidney disease: key roles for calcium and phosphate. Circ Res. 2011 Sep;109(6):697711.

2 Ternacle J, Côté N, Krapf L, Nguyen A, Clavel MA, Pibarot P. Chronic Kidney Disease and the Pathophysiology of Valvular Heart Disease. Can J Cardiol. 2019 Sep;35(9):1195-207.

3 Dobry AS, Ko LN, St John J, Sloan JM, Nigwekar S, Kroshinsky D. Association Between Hypercoagulable Conditions and Calciphylaxis in Patients With Renal Disease: A CaseControl Study. JAMA Dermatol. 2018 Feb; 154(2):182-7.

4 Li Z, Wu J, Zhang X, Ou C, Zhong X, Chen Y, et al. CDC42 promotes vascular calcification in chronic kidney disease. J Pathol. 2019 Dec; 249(4):461-71.

5 Lu ML, Gupta S, Romero-Corral A, Matejková M, De Venecia T, Obasare E, et al. Cardiac Calcifications on Echocardiography Are Associated with Mortality and Stroke. J Am Soc Echocardiogr. 2016 Dec;29(12):1171-8.

6 Aiumtrakul N, Euswas K, Phichedwanichskul K, Rangsin R, Kaewput W, Satirapoj B. Cardiovascular and Renal Outcomes in an Excellent Chronic Kidney Disease Clinic Compared with an Outpatient Clinic in a Primary Care Setting: A Retrospective Cohort Study. Kidney Dis. 2019 Jun;5(3):144-52.

7 Takahashi H, Ishii H, Aoyama T, Kamoi D, Kasuga H, Ito Y, et al. Association of cardiac valvular calcifications and C-reactive protein with cardiovascular mortality in incident hemodialysis patients: a Japanese cohort study. Am J Kidney Dis. 2013 Feb;61(2):254-61.

8 Bortnick AE, Xu S, Kim RS, Kestenbaum B, Ix JH, Jenny NS, et al. Biomarkers of mineral metabolism and progression of aortic valve and mitral annular calcification: The MultiEthnic Study of Atherosclerosis. Atherosclerosis. 2019 Jun;285:79-86.

9 Cadavid JC, DiVietro ML, Torres EA, Fumo $P$, Eiger G. Warfarin-induced pulmonary metastatic calcification and calciphylaxis in a patient with end-stage renal disease. Chest. 2011 Jun;139(6):1503-6.
10 Marwick TH, Amann K, Bangalore S, Cavalcante JL, Charytan DM, Craig JC, et al.; Conference Participants. Chronic kidney disease and valvular heart disease: conclusions from a Kidney Disease: Improving Global Outcomes (KDIGO) Controversies Conference. Kidney Int. 2019 Oct;96(4):836-49.

11 El Hangouche N, Gomez J, Asfaw A, Sreenivasan J, Akhtar T, Teshome M, et al. Impact of pretransplant mitral annular calcification on the incidence of cardiac events after renal transplantation. Nephrol Dial Transplant. 2020 Mar;35(3):526-533.

12 Chen HY, Engert JC, Thanassoulis G. Risk factors for valvular calcification. Curr Opin Endocrinol Diabetes Obes. 2019 Apr;26(2): 96-102.

13 Hisamatsu T, Miura K, Fujiyoshi A, Kadota A, Miyagawa N, Satoh A, et al; SESSA Research Group. Serum magnesium, phosphorus, and calcium levels and subclinical calcific aortic valve disease: A population-based study. Atherosclerosis. 2018 Jun;273:145-52.

14 Cirillo C, Bilancio G, Natale F, Concilio C, Russo MG, Calabrò $\mathrm{P}$, et al. Cardiovascular calcification and subcortical bone demineralization in hypertension. Hypertens Res. 2017 Sep;40(9):825-30.

15 Shuvy M, Abedat S, Beeri R, Danenberg HD, Planer D, Ben-Dov IZ, et al. Uraemic hyperparathyroidism causes a reversible inflammatory process of aortic valve calcification in rats. Cardiovasc Res. 2008 Aug;79(3): 492-9.

16 Ketteler M, Block GA, Evenepoel P, Fukagawa M, Herzog CA, McCann L, et al. Diagnosis, Evaluation, Prevention, and Treatment of Chronic Kidney Disease-Mineral and Bone Disorder: Synopsis of the Kidney Disease: Improving Global Outcomes 2017 Clinical Practice Guideline Update. Ann Intern Med. 2018 Mar;168(6):422-30.

17 Wei X, Wu W, Li L, Lin J, Liu Q, Gan L, et al. Bone Morphogenetic Proteins 2/4 Are Upregulated during the Early Development of Vascular Calcification in Chronic Kidney Disease. BioMed Res Int. 2018 Apr;2018: 8371604.
18 Wen Y, Pan MM, Lv LL, Tang TT, Zhou LT, Wang B, et al. Artemisinin attenuates tubulointerstitial inflammation and fibrosis via the NF- $\kappa \mathrm{B} / \mathrm{NLRP} 3$ pathway in rats with $5 / 6 \mathrm{sub}$ total nephrectomy. J Cell Biochem. 2019 Mar; 120(3):4291-300.

19 Fang Y, Ginsberg C, Sugatani T, MonierFaugere MC, Malluche H, Hruska KA. Early chronic kidney disease-mineral bone disorder stimulates vascular calcification. Kidney Int. 2014 Jan; 85(1):142-50.

20 Ni LH, Tang RN, Lv LL, Wu M, Wang B, Wang FM, et al. A rat model of SHPT with bone abnormalities in CKD induced by adenine and a high phosphorus diet. Biochem Biophys Res Commun. 2018 Apr;498(3):6549.

21 Mulay SR, Eberhard JN, Pfann V, Marschner JA, Darisipudi MN, Daniel C, et al. Oxalateinduced chronic kidney disease with its uremic and cardiovascular complications in C57BL/6 mice. Am J Physiol Renal Physiol. 2016 Apr;310(8):F785-95.

22 Han Y, Lu JS, Xu Y, Zhang L, Hong BF. Rutin ameliorates renal fibrosis and proteinuria in 5/6-nephrectomized rats by anti-oxidation and inhibiting activation of TGF $\beta 1$-smad signaling. Int J Clin Exp Pathol. 2015 May;8(5): 4725-34.

23 Wu M, Tang RN, Liu H, Pan MM, Liu BC. Cinacalcet ameliorates aortic calcification in uremic rats via suppression of endothelial-tomesenchymal transition. Acta Pharmacol Sin. 2016 Nov;37(11):1423-31.

24 Watanabe K, Fujii H, Goto S, Nakai K, Kono K, Watanabe S, et al. Newly Developed Rat Model of Chronic Kidney Disease-Mineral Bone Disorder. J Atheroscler Thromb. 2018 Feb;25(2):170-7.

25 Tani T, Orimo H, Shimizu A, Tsuruoka S. Development of a novel chronic kidney disease mouse model to evaluate the progression of hyperphosphatemia and associated mineral bone disease. Sci Rep. 2017 May;7(1): 2233. 
26 Butcher JT, Tressel S, Johnson T, Turner D, Sorescu G, Jo H, et al. Transcriptional profiles of valvular and vascular endothelial cells reveal phenotypic differences: influence of shear stress. Arterioscler Thromb Vasc Biol. 2006 Jan;26(1):69-77.

27 Sakaue T, Shikata F, Utsunomiya K, Fukae S, Kurata $\mathrm{M}$, Nakaoka $\mathrm{H}$, et al. Proteomicsbased analysis of lung injury-induced proteins in a mouse model of common bile duct ligation. Surgery. 2017 Jun;161(6):1525-35.
28 Wu RN, Yu TY, Zhou JC, Li M, Gao HK, Zhao C, et al. Targeting HMGB1 ameliorates cardiac fibrosis through restoring TLR2-mediated autophagy suppression in myocardial fibroblasts. Int J Cardiol. 2018 Sep;267:156-62.

29 Six I, Maizel J, Barreto FC, Rangrez AY, Dupont S, Slama M, et al. Effects of phosphate on vascular function under normal conditions and influence of the uraemic state. Cardiovasc Res. 2012 Oct;96(1):130-9.

30 Ix JH, Shlipak MG, Katz R, Budoff MJ, Shavelle DM, Probstfield JL, et al. Kidney function and aortic valve and mitral annular calcification in the Multi-Ethnic Study of Atherosclerosis (MESA). Am J Kidney Dis. 2007 Sep;50(3):412-20.
31 Bertazzo S, Gentleman E, Cloyd KL, Chester $\mathrm{AH}$, Yacoub MH, Stevens MM. Nano-analytical electron microscopy reveals fundamental insights into human cardiovascular tissue calcification. Nat Mater. 2013 Jun;12(6):576-83.

32 Roberts WC, Taylor MA, Shirani J. Cardiac findings at necropsy in patients with chronic kidney disease maintained on chronic hemodialysis. Medicine (Baltimore). 2012 May; 91(3):165-78. 